\title{
Association of gene polymorphism at atrial fibrillation: A literature review
}

\author{
Dana Taizhanova ${ }^{1}$, Nazira Bazarova ${ }^{1}$, Elena $Z_{\text {Zholdybaeva }}{ }^{2}$ Akerke Kalimbetova $^{1}$ \\ ${ }^{1}$ Department of Internal Diseases, Medical University of Karaganda, Karaganda city, Republic of Kazakhstan \\ ${ }^{2}$ National Scientific Shared Laboratory of Biotechnology, National Center of Biotechnology, Nur-Sultan city, Republic of Kazakhstan
}

Received: 2020-11-14.

Accepted: $2020-12-08$

This work is licensed under a Creative Commons Attribution 4.0 International License

J Clin Med Kaz 2021; 18(1):19-22

Corresponding author:

Akerke Kalimbetova.

E-mail: akerke.kalimbetova@gmail.com

\begin{abstract}
Atrial fibrillation is the most common persistent heart rhythm disorder, the prevalence of which increases with age. The progressive prevalence of atrial fibrillation is becoming a serious threat to public health throughout the world, as it is associated with high mortality rates. In the review, we analyzed the literature data on the state of knowledge of the genetic nature of atrial fibrillation. To achieve this goal, a systematic search and subsequent analysis of publications and online resources was carried out. All publications are indexed in PubMed, Medline, e-library, Google Scholar. To date, at least 30 loci associated with atrial fibrillation have been identified. At the same time, the research results depend on such factors as ethnicity, concomitant cardiac and extracardiac diseases, inheritance mechanisms, and intergenic interactions. The complexity of etiopathogenesis, the heterogeneity of atrial fibrillation poses the task of researchers to further search for factors that play a leading role in the development of the disease. Significant progress in understanding the genetic basis of atrial fibrillation was achieved with the advent of large-scale genomic GWAS studies, which include genotyping up to a million common variants or single nucleotide polymorphisms. The currently available data on the association of candidate genes with atrial fibrillation in case-control studies are controversial and necessitate more detailed studies on various ethnic groups.
\end{abstract}

Key words: atrial fibrillation, polymorphism, candidate genes, ethnicity, genomic GWAS study

\section{Introduction}

Atrial fibrillation (AF) is the most common persistent heart rhythm disorder, the prevalence of which increases with age [1]. AF prevalence and incidence in a European population depended on the age and was higher in men in prospective study. The high risk of AF developing during lifetime was similar to the epidemiological data in North America. A systematic review of population-based studies of AF published from 1980 to 2010 showed a progressive gradual increase in AF prevalence worldwide [2]. AF prevalence increased by $44 \%$ in the population of Russian Federation from 2010 to 2017 according to statistical analysis conducted by Russian scientists [3]. The results of the study in one of the regions of the Republic of Kazakhstan showed, that AF prevalence in the population over 18 years old was $3.3 \%$ [4].

The progressive AF prevalence is becoming a serious threat to public health worldwide, as it is associated with high mortality rates. AF was an independent risk factor for all-cause mortality in a nationwide long-term case-control study from 1995-2008 in Sweden, and at the same time the differences between the sexes in each age group were statistically significant. AF is independently associated with a twofold increase of death risk from all causes in women and a 1.5-fold increase in men [5]. The main causes of high mortality in patients with AF are the progression of chronic heart failure (CHF) and the development of thromboembolic complications, the most severe of which is the development of cardioembolic cerebral stroke. According to European studies, AF is the cause of ischemic strokes in $1 / 4$ of cases [6].

AF etiopathogenesis of is quite complex, the modern studies confirm both the influence of clinical factors on AF development and the genetic nature of the disease. Clinical factors include organic heart disease and other diseases, the consequence of which may be its structural changes, among them - arterial hypertension (AH), coronary heart disease (CHD), chronic heart failure (CHF), inflammatory myocardial diseases, cardiomyopathies, obesity, diabetes mellitus, thyroid pathology. Real clinical practice shows that 
$32 \%$ of AF cases develop against the background of hypertension. Arterial hypertension is diagnosed in $91 \%$ of patients with AF when analyzing hospitalized patients [7]. Analysis of the structure of combined cardiovascular pathology showed that $81.9 \%$ of hospitalized patients with AF have reliably confirmed coronary artery disease [8]. In addition, the causes of AF include chronic obstructive pulmonary disease and obstructive sleep apnea, chronic renal failure, chronic alcoholism and smoking. AF can be a complication of surgical interventions on the heart and chest in some cases [9].

Clinical conditions cause slow but progressive structural and electrical remodeling of the atrial myocardium, in turn, these pathophysiological mechanisms lead to AF formation. The remodeling process is manifested by fibroblast proliferation and differentiation, by the increased connective tissue deposition and fibrosis. Structural remodeling leads to electrical dissociation of muscle fibers and conduction heterogeneity, which in turn contributes to AF development and maintenance [10].

AF can occur in the absence of organic heart disease in some cases, the cause of AF development is not clinically diagnosed, this AF form is called as isolated or idiopathic. According to published studies, idiopathic AF may be a monogenic syndrome with a classical Mendelian pattern of inheritance. In most cases, these patients have a family history, which suggests a genetic predisposition to AF [11]. AF presence in parents increases the risk of AF in their offspring in 3 times according to the Framingham Study. The risk of AF inheriting increases in individuals who have at least one parent with $\mathrm{AF}$ in history [12]. Isolated AF most often develops at a young age, and the AF prevalence among patients younger than 50 years of age is $0.1 \%$. However, clinically, it does not differ from AF that has arisen against the background of cardiac pathology; therefore, the true incidence of familial AF is unknown [13].

The hereditary predisposition to AF has complex developmental mechanisms. Histologically, atrial myocardial fibrosis is detected at isolated AF, which leads to the formation of fibrous cardiomyopathy, which is assumed to have a genetic predisposition. In particular, AF can develop at certain genotypes of genes polymorphism operating in the renin-angiotensinaldosterone system (RAAS), which are involved into the regulation of myocardial fibrosis [14].

Further identification and study of various monogenic forms of AF makes it possible to study the molecular mechanisms of not only familial AF cases, but also its polygenic variants. Despite the fact that monogenic forms are rare, the study of their genetic nature is an urgent practical task. An important factor in AF genesis is potassium channel mutations, which induce triggering activity in the atria. The hypothesis that different forms of AF are based on common molecular mechanisms was made possible by the data on the formation of similar phenotypes of ion channels resulting from electrophysiological remodeling of the myocardium in patients with nonfamilial forms of chronic $\mathrm{AF}$ and in individuals with monogenic AF forms [15].

The variability of AF predisposition genes is represented by single nucleotide polymorphism in most cases. These genetic variants affect the risk of $\mathrm{AF}$ development in combination with other factors, as they have low penetrance. According to studies of $\mathrm{AF}$ genetic nature, the occurrence of $\mathrm{AF}$ is facilitated by the combined carriage of certain variants of several candidate genes [16-18]. In most cases, the onset of AF is facilitated by certain combinations of polymorphisms of different genes. Therefore, the screening of susceptibility genes and the study of their polymorphism is currently becoming an important direction in the study of AF.

Thus, at present, molecular studies of AF are focused on identifying genes whose mutations lead to arrhythmias (such arrhythmias are inherited according to the classical Mendelian pattern) and studying the polymorphism of various genes, the so-called candidate genes. The verification of genetic variants and the search for genes associated with AF have been carried out for many years. Analysis of relationships and sequencing of candidate genes revealed multiple polymorphisms in both familial forms and sporadic cases of AF [19]. As a result of studying the relationship between the polymorphism of the RAAS genes and the development of AF, N. P. Topal et al. (2001) genotyped the following polymorphisms: I/D polymorphism of the ACE gene; M235T, A-20C, and G-6A polymorphisms of the AGTR gene [20]. R. Ma et al. in their meta-analysis investigated the relationship of I/D polymorphism of the ACE gene in association with AF. They traced the relationship between ethnicity and I/D polymorphism of the ACE gene and concluded that individuals with the DD genotype of ACE gene are more at risk of developing AF [21]. Jiang M. H. et al. (2010) conducted a study of rs4343 (2350 G/A) polymorphism of ACE gene in patients with hypertension in the Chinese population. It was found that $2350 \mathrm{G} / \mathrm{A}$ polymorphism is associated with AF and $\mathrm{A}$ allele determines the increased risk of $\mathrm{AF}$ in this group of patients [22]. Further, H. Wang et al. (2015), using a metaanalysis, found that M235T polymorphism of AGT gene may be associated with an increased risk of AF development in the Asian population [23]. B. Chalazan et al. (2018) for the first time established a relationship between chromosome 4q25 SNP and AF development in Hispanics [24]. In the same year, a new genetic marker of AF was verified in the Japanese population HCN4 gene, the single nucleotide polymorphism of rs7164883 HCN4 gene [25]. A comprehensive meta-analysis has shown that rs1799998 polymorphism may serve as a potential biological marker of AF in East Asian patients with AF [26].

Most of the earlier studies have focused on specific candidate genes associated with AF, while studying of AF genetic basis in the general population remains more challenging. To date, a significant achievement is the verification in a number of associative studies of case-control design, common genetic variants that are most common in populations of patients with AF in comparison with healthy controls. Significant progress in AF genetic basis understanding has been achieved with the advent of genome-wide association studies (GWAS). GWAS is a large-scale genomic study that includes genotyping of up to a million common variants or single nucleotide polymorphisms (SNPs) and compares their frequency in AF patients and in control groups [27]. As a result of these studies, it is possible to identify genes whose participation in AF development has not been previously established in contrast to case-control studies. To date, more than four large GWAS have been performed in cohorts of patients with AF [28-31].

A meta-analysis of GWAS for AF identified six new susceptibility loci or about 10 probable candidate genes, showed that atrial fibrillation has multiple genetic associations, and sets new targets for further research [32]. Further, a systematic review identified nine noncoding SNPs associated with an increased risk of AF development [33]. Large-scale genotyping in Europeans and Japanese identified new AF risk loci in the NEURL, TBX5, CAND2, GJA1, and CUX2 genes or near them [34]. At least 30 loci associated with AF have been identified in presence. 
A multiethnic study of the genomic association for atrial fibrillation with more than half a million participants, including 65446 with AF from more than 50 studies, confirms the role of PRRX1, TBX5 and KCNJ5 genes in the occurrence of AF, with a sample of $84.2 \%$ European, 12.5\% Japanese, 2\% African American, and 1.3\% Brazilian and Hispanic [35].

It should be noted that there are significant racial and ethnic differences in AF prevalence, so it is important to take population characteristics into account when conducting genetic studies. In particular, African Americans and Japanese have been found to be less prone to AF than Europeans [36]. In particular, according to the results of the study of Russian scientists, no statistically significant data were found on the relationship between the $\mathrm{A} / \mathrm{C}$ polymorphism of the AGTR1 gene and the development of AF in any group of patients. The research results contradict the data of foreign studies. Thus, Chinese scientists have obtained data that $\mathrm{C}$ allele of AGTR1 gene significantly increases the risk of AF developing. As a result of the study of PITX2 and NEURL1 SNP in Hungarian patients with AF, determined by quantitative PCR, no significant association was found between SNP rs2595104 and rs6584555 and AF [37]. The obtained results may be due to genetic characteristics of the population, depending on climatic conditions, geography of residence, and confirm that $\mathrm{AF}$ is a heterogeneous disease. It should be noted that genetic predictors of AF can be combined with genetic predictors of other diseases, which may complicate the choice of candidate genes for researchers [38].

According to ESC clinical guidelines for AF, (2017), routine testing to identify common variants of AF-associated genes cannot be universally recommended at present [39]. However, the results of the study published by American scientists indicate that both the incidence and prevalence of AF are growing at a steady pace in the US population and indicate that the public health burden of AF may reach high levels by 2030 [40]. Mortality from cerebrovascular diseases in the Republic of Kazakhstan for 12 months of 2019 amounted to 58.9 per 100 thousand of population according to the Committee on Statistics of the Ministry of National Economy of the Republic of Kazakhstan, while the cause of death in $30 \%$ of cases at vascular brain damage is cardioembolic strokes against the background of AF. This indicator has the tendency to decrease in comparison with the previous year, but remains high. Assessment of the polymorphism of candidate genes associated with AF, depending on ethnicity, would expand the possibility of this disease preventing. Screening of candidate genes will allow identification of groups of individuals at the preclinical level that have a genetic substrate for AF development in a particular population. Thus, it will be possible to implement personalized prevention of AF based on ethnicity.

\section{Conclusion}

Currently, significant progress has been achieved in genetic substrate identifying that underlying AF development. At the same time, the research results depend on such factors as ethnicity, concomitant cardiac and extracardiac diseases, inheritance mechanisms and intergenic interactions. The complexity of etiopathogenesis, AF heterogeneity poses the task to further search for factors that play a leading role in the development of the disease. Currently available data on the associations of candidate genes with AF are contradictory and necessitate more detailed studies on various ethnic groups. To date, gene polymorphism at AF has not been studied in populations living in Kazakhstan. It is not enough to simply project the data of foreign studies onto the large population of our state. Assessment of the polymorphism of candidate genes associated with AF depending on ethnicity will open up new possibilities for a personalized approach in the prevention and treatment of patients with AF.

Disclosures: There is no conflict of interest for all authors.

\section{References}

1. Benjamin, Emelia J et al. Heart Disease and Stroke Statistics-2019 Update: A Report From the American Heart Association. Circulation. 2019; 139(10:e56-e528. doi:10.1161/CIR.0000000000000659.

2. Chugh, Sumeet S., et al. "Worldwide epidemiology of atrial fibrillation: a Global Burden of Disease 2010 Study." Circulation. 2014; 129(8):837-847. doi: 10.1161 / CIRCULATIONAHA.113.005119

3. Kolbin A. S., Mosikyan A. A., Tatarskiy B. A. Socio-economic burden of atrial fibrillation in Russia: dynamics for 7 years (2010-2017). Bulletin of arrhythmology. 2018; 92:42-48.

4. Akpanova D. M. et al. Prevalence and general risk factors for the development of atrial fibrillation. Bulletin of the Kazakh National Medical University. 2016; 2:70-75.

5. Andersson, Tommy, et al. All-cause mortality in 272186 patients hospitalized with incident atrial fibrillation 1995-2008: a Swedish nationwide long-term case-control study. European heart journal. 2013; 34(14):1061-1067. https://doi.org/10.1093/eurheartj/ehs469

6. Fisher, Marc, Joseph A. Hill. Ischemic stroke mandates cross-disciplinary collaboration. (2018):103-105. https://doi.org/10.1161/ CIRCULATIONAHA.117.032903

7. Suslov A. S. Assessment of the nosological affiliation of atrial fibrillation in real clinical practice. Practical medicine. 2014; 6(82):114117.

8. Trigub A. V. Clinical characteristics of patients with various forms of atrial fibrillation. Innovations in medicine. 2017; 144-148.

9. Soloviyov O. V., Lukyanova I. Yu. Clinical conditions associated with atrial fibrillation not associated with valvular heart disease. Juvenis Scientia. 2019; 7:8-13.

10. January, Craig T., et al. 2014 AHA/ACC/HRS guideline for the management of patients with atrial fibrillation: a report of the American College of Cardiology/American Heart Association Task Force on Practice Guidelines and the Heart Rhythm Society. Journal of the American College of Cardiology. 2014; 64(21):e1-e76. 
11. Marcus, Gregory M., et al. A first-degree family history in lone atrial fibrillation patients. Heart Rhythm. 2008; 5(6):826-830. https:// doi.org/10.1016/j.hrthm.2008.02.016

12. Ellinor, Patrick T., et al. Familial aggregation in lone atrial fibrillation. Human genetics. 2005; 118(2):179-184. DOI: 10.1007/s00439005-0034-8

13. Chen, Lin Y., et al. Lone atrial fibrillation: influence of familial disease on gender predilection. Journal of cardiovascular electrophysiology. 2008; 19.8:802-806. https://doi.org/10.1111/j.1540-8167.2008.01126.x

14. Anumonwo, Justus MB, and Jérôme Kalifa. Risk factors and genetics of atrial fibrillation. Heart failure clinics. 2016; 12(2):157-166. DOI:https://doi.org/10.1016/j.hfc.2015.08.013

15. Mahida, Saagar, et al. Monogenic atrial fibrillation as pathophysiological paradigms. Cardiovascular research. 2011; 89(4):692-700. https://doi.org/10.1093/cvr/cvq381

16. Fatkin, Diane, et al. Genetics of atrial fibrillation: state of the art in 2017. Heart, Lung and Circulation. 2017; 26(9):894-901. https:// doi.org/10.1016/j.hlc.2017.04.008

17. Chalazan, Brandon, et al. Genetic modulation of atrial fibrillation risk in a Hispanic/Latino cohort. PloS one. 2018; $13(4)$ :e0194480. https://doi.org/10.1371/journal.pone.0194480

18. A. B. Kuskayeva, S. Yu. Nikulina, A. A. Chernova, N. V. Aksyutina. Genetic predictors of atrial fibrillation. Ration. pharmacotherapy in cardiology. 2016; 12(3):331-336. https://doi.org/10.20996/1819-6446-2016-12-3-331-336

19. Mahida, Saagar. Genetic discoveries in atrial fibrillation and implications for clinical practice. Arrhythmia \& electrophysiology review. 2014; 3.2:69-75 doi: 10.15420/aer.2014.3.2.69

20. Topal, Nurdan Papila, et al. Polymorphisms of the angiotensin-converting enzyme and angiotensinogen gene in patients with atrial fibrillation. Journal of the Renin-Angiotensin-Aldosterone System. 2011; 12(4): 549-556. https://doi.org/10.1177/1470320311399605

21. Ma, Runwei, et al. Angiotensin-converting enzyme insertion/deletion gene polymorphisms associated with risk of atrial fibrillation: A meta-analysis of 23 case-control studies. Journal of the Renin-Angiotensin-Aldosterone System. 2015; 16(4):793-800. https://doi. org/10.1177/1470320315587179

22. Wang, Qun-shan, et al. Angiotensinogen polymorphisms and acquired atrial fibrillation in Chinese. Journal of Electrocardiology. 2010; 43(4):373-377. https://doi.org/10.1016/j.jelectrocard.2009.09.009

23. Wang, HuiXuan, et al. The M235T polymorphism in the angiotensinogen gene and atrial fibrillation: A meta-analysis. Journal of the Renin-Angiotensin-Aldosterone System. 2015; 16.3:647-652. https://doi.org/10.1177/1470320313513482

24. Chalazan, Brandon, et al. Genetic modulation of atrial fibrillation risk in a Hispanic/Latino cohort. PloS one. 2018; 13(4):e0194480. https://doi.org/10.1371/journal.pone.0194480

25. Nakano, Yukiko, et al. HCN4 gene polymorphisms are associated with occurrence of tachycardia-induced cardiomyopathy in patients with atrial fibrillation. Circulation: Genomic and Precision Medicine. 2018; 11(7):e001980. https://doi.org/10.1161/CIRCGEN.117.001980

26. Wang, Xiaoxu, Yingzheng Li, and Qiulai Li. A comprehensive meta-analysis on relationship between CYP11B2 rs1799998 polymorphism and atrial fibrillation. Journal of Electrocardiology. 2019; 52:101-105. https://doi.org/10.1016/j.jelectrocard.2018.11.009

27. Manolio, Teri A. Genomewide association studies and assessment of the risk of disease. New England journal of medicine. 2010; 363(2):166-176. DOI: 10.1056/NEJMra0905980

28. Gudbjartsson D.F., Arnar D.O., Helgadottir A., Gretarsdottir S., Holm H., Sigurdsson A. et al. Variants conferring risk of atrial fibrillation on chromosome 4q25. Nature. 2007; 448(7151):353-7. DOI: 10.1038/nature06007

29. Benjamin, Emelia J., et al. Variants in ZFHX3 are associated with atrial fibrillation in individuals of European ancestry. Nature genetics. 2009; 41(8):879-881. DOI: 10.1038/ng.416

30. Ellinor, Patrick T., et al. Common variants in KCNN3 are associated with lone atrial fibrillation. Nature genetics. 2010; 42(3):240-244. DOI: $10.1038 /$ ng.537

31. Ellinor P.T., Lunetta K.L., Albert C.M., Glazer N.L., Ritchie M.D., Smith A.V. et al. Meta-analysis identifies six new susceptibility loci for atrial fibrillation. Nat. Genet. 2012; 44(6):670-5. DOI: 10.1038/ng.2261

32. Olesen, Morten S., et al. Atrial fibrillation: the role of common and rare genetic variants. European Journal of Human Genetics. 2014; 22(3):297-306. https://doi.org/10.1038/ejhg.2013.139

33. Sinner, Moritz F., et al. Integrating genetic, transcriptional, and functional analyses to identify 5 novel genes for atrial fibrillation. Circulation. 2014; 130(15):1225-1235. https://doi.org/10.1161/CIRCULATIONAHA.114.009892

34. Huang, Henry, and Dawood Darbar. Genetic heterogeneity of atrial fibrillation susceptibility loci across racial or ethnic groups. 2017; 2595-2598. https://doi.org/10.1093/eurheartj/ehx289

35. Roselli C. et al. A multi-ethnic study of the genomic association for atrial fibrillation. Nature Genetics. 50(9):1225-1233. https://doi. org/10.1038/s41588-018-0133-9

36. Huang, Henry, Dawood Darbar. Genetic heterogeneity of atrial fibrillation susceptibility loci across racial or ethnic groups. 2017; 25952598. https://doi.org/10.1093/eurheartj/ehx289

37. Szirák, Krisztina, et al. PITX2 and NEURL1 SNP polymorphisms in Hungarian atrial fibrillation patients determined by quantitative real-time PCR and melting curve analysis. Journal of biotechnology. 2019; 299:44-49. https://doi.org/10.1016/j.jbiotec.2019.04.022

38. Kotecha, Dipak, et al. Integrating new approaches to atrial fibrillation management: the 6th AFNET/EHRA Consensus Conference. Ep Europace. 2018; 20(3):395-407. https://doi.org/10.1093/europace/eux318

39. Kirchhof, Paulus, et al. ESC recommendations for the treatment of patients with atrial fibrillation, developed in conjunction with EACTS. Russ J Cardiol. 2017; 7(147):7-86 http://dx.doi.org/10.15829/1560-4071-2017-7-7-86.

40. Colilla, Susan, et al. Estimates of current and future incidence and prevalence of atrial fibrillation in the US adult population. The American journal of cardiology. 2013; 112(8):1142-1147. https://doi.org/10.1016/j.amjcard.2013.05.063 